\title{
Protein prenylation and human diseases: a balance of protein farnesylation and geranylgeranylation
}

\author{
XU Na, SHEN Ning, WANG XiuXing, JIANG Shan, XUE Bin \& LI ChaoJun* \\ Ministry of Education Key Laboratory of Model Animals for Disease Study, Model Animal Research Center and Medical School of Nanjing \\ University, National Resource Center for Mutant Mice, Nanjing 210061, China
}

Received October 27, 2014; accepted January 23, 2015

\begin{abstract}
The protein prenylation is one of the essential post-translational protein modifications, which extensively exists in the eukaryocyte. It includes protein farnesylation and geranylgeranylation, using farnesyl pyrophosphate (FPP) or geranylgeranyl pyrophosphate (GGPP) as the substrate, respectively. The prenylation occurs by covalent addition of these two types of isoprenoids to cysteine residues at or near the carboxyl terminus of the proteins that possess CaaX motif, such as Ras small GTPase family. The attachment of hydrophobic prenyl groups can anchor the proteins to intracellular membranes and trigger downstream cell signaling pathway. Geranylgeranyl biphosphate synthase (GGPPS) catalyzes the synthesis of 20-carbon GGPP from 15-carbon FPP. The abnormal expression of this enzyme will affect the relative content of FPP and GGPP, and thus disrupts the balance between protein farnesylation and geranylgeranylation, which participates into various aspects of cellular physiology and pathology. In this paper, we mainly review the property of this important protein post-translational modification and research progress in its regulation of cigarette smoke induced pulmonary disease, adipocyte insulin sensitivity, the inflammation response of Sertoli cells, the hepatic lipogenesis and the cardiac hypertrophy.
\end{abstract}

protein prenylation, GGPP, FPP, biological function regulation

Citation: $\quad \mathrm{Xu} \mathrm{N}$, Shen N, Wang XX, Jiang S, Xue B, Li CJ. Protein prenylation and human diseases: a balance of protein farnesylation and geranylgeranylation. Sci China Life Sci, 2015, 58: 328-335, doi: 10.1007/s11427-015-4836-1

The post-translational lipid modification of proteins has been recognized as a key process for the regulation of protein localization and physiological function. The protein prenylation is one of the most important modifications critical for the membrane association of a plethora of signaling proteins with fundamental roles in cell biology, including cell growth, differentiation, proliferation and protein trafficking [1]. The two types of isoprenoids, farnesyl pyrophosphate (FPP) and geranylgeranyl pyrophosphate (GGPP), are synthesized by the cellular mevalonate pathway. Protein prenylation occurs by the addition of them to a cysteine residue located at or near the carboxyl terminal end of the protein [2]. A series of signaling pathway will be altered

*Corresponding author (email: licj@nju.edu.cn) once this modification is disrupted, which accounts for the pathological progression of many diseases including neurodegenerative disorders and cancers.

\section{The sources of FPP and GGPP}

The isoprenoids are all derived from the common fivecarbon $\left(\mathrm{C}_{5}\right)$ building unit isopentenyl diphosphate (IPP) and its isomer dimethylallyl diphosphate (DMAPP). The headto-tail or head-to-head addition of $\mathrm{C}_{5}$ units forms the building blocks of the tens of thousands of more complex isoprenoids [3]. Isoprenoids are synthesized exclusively through the mevalonate (MVA) pathway in animals, fungi and archaebacteria. Besides, plants and microbes can also 
use 2-C-methyl-D-erythritol-4-phosphate (MEP) pathway for isoprenoid synthesis $[1,4,5]$. In the MVA pathway, firstly acetyl-CoA is converted to 3-hydroxy-3-methylglutaryl coenzyme A (HMG-CoA) by HMG-CoA synthase, and then to mevalonate by HMG-CoA reductase. Subsequently, the FPP, which is synthesized from IPP by FPP synthase (FPPS), can be converted through four different pathways via a number of enzyme steps. One is to synthesize GGPP by GGPP synthase (GGPPS). One is to farnesylate the proteins containing $\mathrm{CaaX}$ motif by farnesyltransferase (FTase). The other is to produce cholesterol by a series of processes. Additionally, FPP can also be used to synthesize Heme A and dolichol, etc. GGPP, which is derived directly from FPP, can make the proteins containing CaaX motif geranylgeranylated by geranylgeranyltransferase (GGTase) and be also used to synthesize ubiquinone (Figure 1).

\section{Protein prenylation}

Protein prenylation, which occurs shortly after translation, is irreversible during the lifetime of the protein [2]. It contains protein farnesylation and geranylgeranylation that is catalyzed by FTase or GGTase (GGTase I and II), respectively. FTase and GGTase I are both heterodimers and consist of two subunits, $\alpha$ and $\beta$. The $\alpha$ subunit is encoded by the same gene, FNTA. The $\beta$ subunit is encoded by FNTB and $P G G T I B$ respectively [6]. Both of these two transferases recognize proteins that possess a $\mathrm{CaaX}$ motif at their C-terminal end, where $\mathrm{C}$ is the cysteine that becomes prenylated, $\mathrm{a}$ is an aliphatic amino acid and $\mathrm{X}$ is any other residue. The nature of the $X$ residue determines whether a protein will be farnesylated or geranylgeranylated. Proteins

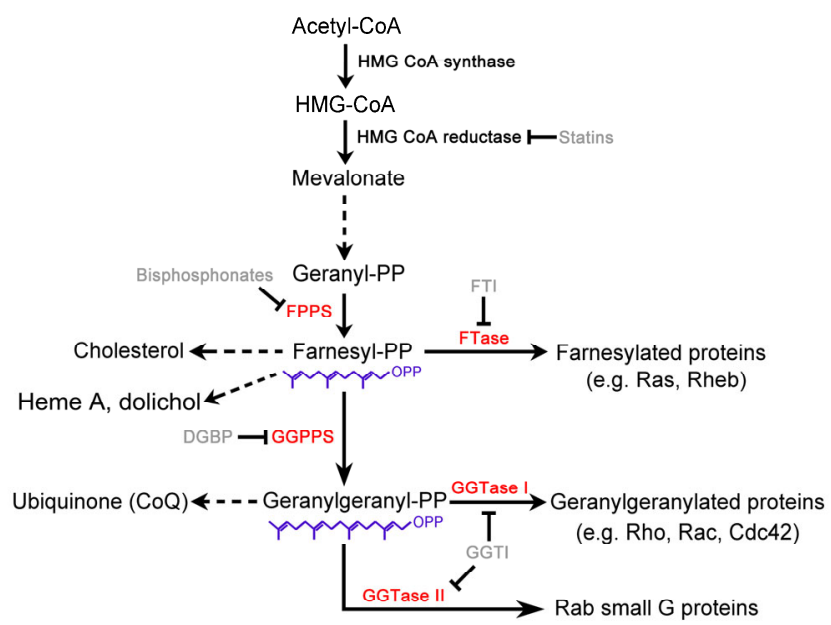

Figure 1 (color online) The mevalonate pathway. Mevalonate is the precursor of FPP and GGPP, which are the substrate for prenylation enzymes. In addition to prenylation, FPP can produce cholesterol, Heme A and dolichol via a series of steps. GGPP can synthesize ubiquinone. The specific inhibitors targeting corresponding enzymes are indicated in grey font. ending in serine, methionine, alanine or glutamine become farnesylated, while proteins ending in leucine or isoleucine become geranylgeranylated [2,7]. However, there are a few exceptions that proteins can alternatively undergo either type of modification. When $\mathrm{X}$ is phenylalanine, the proteins can be either farnesylated or geranylgeranylated [8]. The alternative prenylation can occur under situations of limited isoprenoid availability. For example, K-Ras can be geranylgeranylated in cells treated with farnesyltransferase inhibitors (FTIs) [9].

The typical substrates that are farnesylated by FTase include many members of Ras superfamily of small GTPases (H-Ras, K-Ras, N-Ras, Ras2, Rap2, RhoB, RhoE, Rheb, TC10, TC21), as well as the nuclear lamina proteins lamin $\mathrm{A}$ and $\mathrm{B}$, the kinetochore proteins CENP-E and CENP-F, cGMP phosphodiesterase $\alpha, \gamma$ subunit variants of $G$ proteins, DnaJ heat-shock protein homologs, rhodopsin kinase, the peroxisomal membrane proteins Pex19 and PxF and so on. GGTase I preferentially geranylgeranylates some of the other small GTPases (Rac1, Rac2, RalA, Rap1B, RhoA, RhoB, RhoC, Cdc42, Rab8, Rab11, Rab13), as well as some $\gamma$ subunit variants of $\mathrm{G}$ proteins, cGMP phosphodiesterase $\beta$ and the plant calmodulin CaM53 [6]. GGTase II, also called Rab geranylgeranyltransferase, transfers one or two molecules of geranylgeranyl to Rab family which contains $\mathrm{CC}$, CAC, CCX or CCXX motifs. The prenylation by GGTase II requires the participation of the Rab escort protein (REP), an auxiliary protein involved in the recognition of Rab by GGTase II [2].

The largest families of prenylated proteins are the intracellular GTP-binding proteins involved in signaling transduction. Protein prenylation is essential for their GDP/GTP binding changes and GTPase activity happening on the cell membrane because it directly makes the proteins associated with membrane compartments [1]. Either a farnesyl or a geranylgeranyl group attachment to a cysteine at or near the C-terminal end is through a thioether linkage (-C-S-C-), following further post-prenylation processing. Briefly, the three C-terminal amino acids ( $\mathrm{aaX}$ ) are then cleaved by the endoprotease Rce-1, and the remaining C-terminal prenylated cysteine residue is carboxymethylated by an S-adenosylmethione (SAM)-dependent protein carboxymethylase [2]. This group of modifications increases the hydrophobicity of the protein and facilitates its interaction with membranes (Figure 2).

\section{Protein prenylation and diseases}

Given the ubiquitous nature of the protein prenylation and its roles in cellular biology, defects in isoprenoid biosynthesis or regulation can cause lots of diseases, such as cancer, cardiovascular diseases, neurodegenerative disorders and metabolic diseases. The abnormal expression or even activity of enzymes involved in the MVA pathway can lead to 


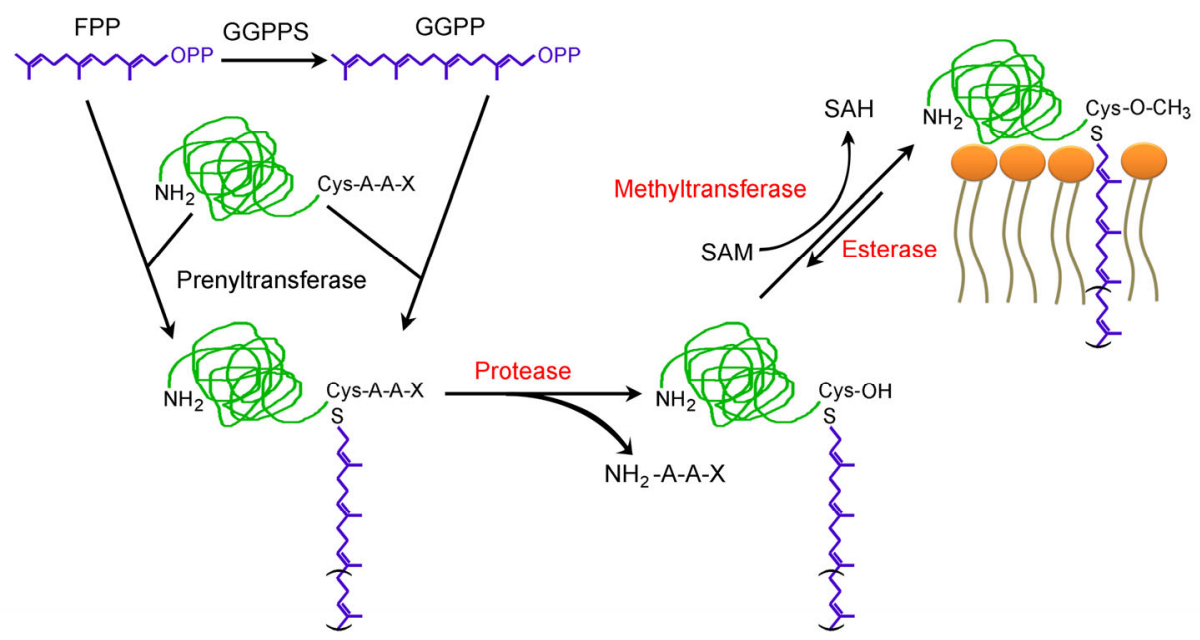

Figure 2 (color online) The post-prenylation processing. Following prenylation, the prenylated protein protease removes the three C-terminal amino acids $(\mathrm{aaX})$, and the remaining cysteine residue is carboxymethylated by a specific methyltransferase. This methylation is reversible due to its esterase activity. The modification increases the hydrophobicity of the protein and facilitates its interaction with membranes. SAM, S-adenosyl-methionine; SAH, S-adenosyl-homocysteine.

impaired protein prenylation. Mutations within the Ras family could be identified in as many as $10 \%-15 \%$ of all human cancers. Rational therapies that target the Ras prenylation by genetic methods and pharmacological intervention may repress Ras-mediated malignant transformation [10]. FTIs and GGTIs have been recently developed as potential anticancer drugs because of their inhibition on Ras and Rho family respectively [7]. The research by Mijimolle utilizing FTase $\beta$ subunit knockout mouse has provided that farnesylated proteins are not required for the initial malignant cellular transformation but are critical for tumor progression and maintenance [11]. It is also reported that ablation of GGTase I $\beta$ subunit could inhibit K-Ras induced lung tumour formation [12], but activate inflammatory response in macrophages through Rho signaling [13]. Mutations in the GGTase II gene caused Hermansky-Pudlak syndrome, characterized by disorders of pigmentation, prolonged bleeding, and ceroid deposition, often accompanied by severe fibrotic lung disease and colitis [14]. On the other hand, mutations in REP-1 could lead to retinal degeneration associated with a defect in the prenylation of Rab27a $[15,16]$. It is suggested that GGPP stimulated PPAR $\gamma$ expression and adipogenesis and thus inhibited osteoblast mineralization and differentiation [17]. Direct inhibition of GGPPS by digeranyl bisphosphonate (DGBP) resulted in depletion of GGPP and impaired protein geranylgeranylation in MC3T3-E1 preosteoblast cells but accumulated FPP level. Therefore, DGBP treatment did not lead to increased osteoblast differentiation [18].

\subsection{The Ras prenylation- and cigarette smoke-induced pulmonary disease}

The long-term stress of low intensity stimulation by the hazardous materials is the cause of some chronic diseases, for instance, long-term cigarette smoke stimulation leads to the COPD (chronic obstructive pulmonary disease), eventually the loss of function of pulmonary. Cigarette smoke is the reason for $80 \%-90 \%$ of patients with COPD, nevertheless only $10 \%-15 \%$ of smokers exhibit obviously clinical obstructive pulmonary. Thus the variation of individual genes expression leads to the different response to cigarette smoke. In addition, the mechanism by which long-term stimulation of cigarette smoke leads to severe clinical diseases is still unknown. In our previous study, cigarette smoke induced the early growth response gene (Egr-1) expression through MAPK signaling and regulated the metalloprotease activity and the inflammation in pulmonary [19-21]. We revealed that Egr-1 could bind to the promoter of GGPPS gene in a cigarette smoke dependent way through ChIP and identified GGPPS as one of the target genes of Egr-1 [22]. Furthermore, both Egr-1 and its target genes GGPPS and HO-1 were increasingly expressed in the lung from the cigarette smoke induced COPD mice. GGPPS is the enzyme that prenylates and activates Ras and re-activates the MAPK/Erk1/2, sequentially activates Egr-1 expression in a positive feedback way $[23,24]$. The constant activated Egr-1 by long-term smoke enhances the inflammation in pulmonary and over-activates the metalloprotease, eventually aggravates the impairment of lung tissue. Together, we declared that the activity of Egr-1/GGPPS/Ras pathway is highly relevant to cigarette smoke-induced pulmonary diseases.

\subsection{Protein prenylation and insulin resistance}

Obesity is the primary cause of insulin resistance [25]. GGPPS is highly expressed in the liver, muscle and adipose 
tissue from $o b / o b$ mice with extreme insulin resistance and hyperinsulinemia [26]. It is also reported that insulin can activate the geranylgeranyl transferase and induce Rab geranylgeranylated in 3T3-L1 preadipocytes. Inhibiting the geranylgeranyl transferase activity can down-regulate the phosphorylation of MAPK pathway without affecting the PI3K/AKT pathway in 3T3-L1 preadipocytes [27].

In our study, the positive feedback way of MAPK/Egr-1/ GGPPS/Ras also exerts a primary role in other stress such as hyperinsulinism of type 2 diabetes. In this case, insulin can trigger both the opposite pathway: PI3K/AKT and MAPK. PI3K/AKT promotes the glucose uptake that is the positive function of insulin while MAPK can induce insulin resistance by activating gene expression that is the negative function of insulin. We found that the long-term insulin stimulation turned the transient elevation of Egr-1 constantly expressed so as to make GGPPS constantly activated. GGPPS further activated K-Ras by enhancing its geranylgeranylation and constantly activated the Ras/MAPK/Erk1/2 signaling which could phosphorylate IRS-1 at Ser612, eventually inhibited the insulin signaling and promoted the insulin resistance [28]. Furthermore, Egr-1 could activate both the PI3K/AKT and MAPK pathway. Egr-1 dephosphorylated PIP3 to PIP2 and inhibited the activation of AKT by PI3K through PTEN. The highly expressed Egr-1 in adipose tissue not only led to adipose insulin resistance but also enhanced the inflammation cytokines secretion such as TNF- $\alpha$ and IL-6, finally inhibited the systemic insulin sensitivity. Over-expression of Egr-1 in adipose tissue in mice could result in systemic insulin resistance while loss of function of adipocytes Egr-1 could improve the insulin sensitivity in $d b / d b$ mice [29]. Taken together, our study revealed that Egr-1, as an important reg- ulator, could simultaneously regulate both PI3K/AKT and MAPK signaling pathway (Figure 3). This makes Egr-1 a new potential pharmaceutical target for insulin resistance.

\subsection{The Ras prenylation and male infertility}

Mumps commonly affects children 5-9 year of age, and can lead to permanent adult sterility in certain cases. Mumps infection results in proliferation of macrophages and progressive degeneration of the seminiferous tubules, exhibiting a significant spermatogonia loss and occasionally, Sertoli cell-only syndrome [30-32]. A physical barrier, the so-called blood-testis barrier (BTB), forms between Sertoli cells and allows for these cells to control and regulate the environment of the developing germ cells. More importantly, in addition to the BTB, Sertoli cells can secrete immunomodulatory factors that create an effectively immune-privileged environment [33,34].

We observed that GGPPS expression in the testis of infertile patients with a history of mumps infection was significantly lower compared with that in noninfected infertile patients, because of the enhanced methylation level of the GGPPS promoter after mumps infection. Given that GGPPS was primarily observed in the cytoplasm of Sertoli cells and spermatogonia, and Sertoli cells played a central role in the regulation of immune privilege during adulthood, we hypothesized that GGPPS regulated protein prenylation in Sertoli cells was crucial for virus infection. To further study the function of GGPPS in immune response, we constructed Sertoli cell-specific GGPPS knockout mice. The Sc$G G P P S^{-/-}$mice exhibited adult infertility. Surprisingly, although the GGPPS gene was deleted in Sertoli cells rather than in spermatogonia, the number of Sertoli cells in testic-

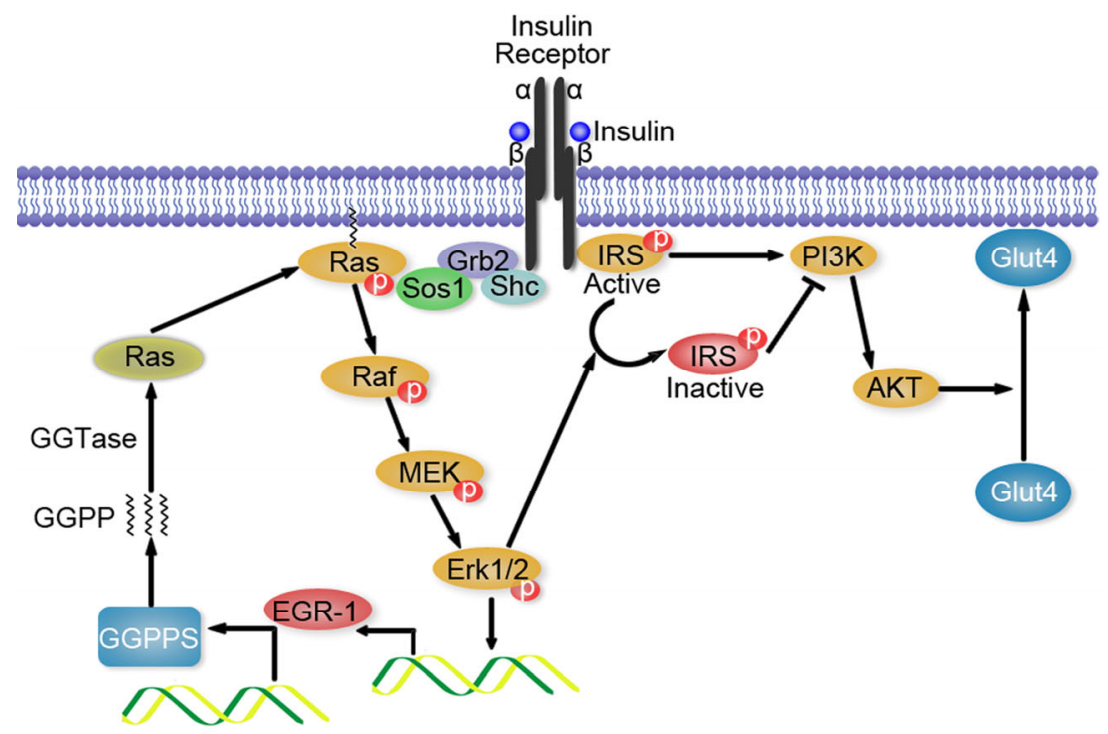

Figure 3 (color online) GGPPS regulates insulin sensitivity in adipocyte [28]. Egr-1 is greatly increased in hyperinsulinism and in insulin resistance through activation of the Erk1/2 MAPK pathway. The highly expressed Egr-1 can reactivate Erk1/2 by triggering GGPPS transcription and promoting Ras membrane association. The sustained activation of Erk1/2 can phosphorylate IRS-1 on Ser612 and inhibit its activity. 
ular tubules was unaltered, whereas the number of spermatogonia was significantly decreased at postnatal $5 \mathrm{~d}$. Because there is no BTB between Sertoli cells until 3 weeks, the spermatogonia loss at this stage was induced by increased cytokines and chemokines release and seminiferous tubule macrophage invasion. Further analysis showed that GGPPS deletion in Sertoli cells increased H-Ras farnesylation and constitutively activated MAPK Erk1/2, which was responsible for $\mathrm{NF}-\kappa \mathrm{B}$ activation and inflammatory reaction, and thus for the augmentation of cytokines (IL-6, TNF- $\alpha$ ) and chemokines (G-CSF, CCL2 (MCP-1), CCL3 (MIP-1 $\alpha$ ), CXCL2 (MIP-2 $\alpha$ )). These inflammatory factors then bound to the receptors on spermatogonia and activated cell apoptosis signaling pathway, leading to a long-term effect on adult infertility [35,36]. Macrophages could be recruited by chemokines, easily invade the testicular tubules and attack developing spermatogonium cells, resulting in spermatogonia apoptosis [37].

Our results indicated that protein prenylation in Sertoli cells was critical for regulating the immune response in testis before puberty (Figure 4). Our data provided a novel mechanism by which mumps infection during childhood results in adult sterility.

\subsection{Protein prenylation and nonalcoholic fatty liver disease}

Non-alcoholic fatty liver disease (NAFLD) represents a histological spectrum of liver disease characterized by ex- cess fat accumulation in the liver without a history of alcohol abuse [38]. As economy grows and the life style changes, NAFL increased gradually and has emerged as one of the most prevalent chronic liver disease in the past few decades. The incidence of NAFL is about $20 \%$ in Chinese but reaches as high as $75 \%$ in obese people [39]. It ranges from simple steatosis (hepatic steatosis, non-alcoholic fatty liver, NAFL) to non-alcoholic steatohepatitis (NASH), advanced liver fibrosis, cirrhosis and ultimate hepatocellular carcinoma (HCC) [40,41]. Extensive research and clinical studies have been performed to investigate the pathogenesis of NALFD, although the precise cellular networks are still not fully elucidated.

Simple steatosis can be reversed. Therefore, understanding the mechanism of TG accumulation would help to identify therapeutic interventions for hepatic steatosis. Our research demonstrated that GGPPS was up-regulated in the liver of obesity-associated hepatic steatosis mice models. Cellular over-expression of GGPPS resulted in massive TG accumulation in primary hepatocytes, and elevated lipid droplets marker genes as ADRP, Perlipin, aP2 and C/EBP $\alpha$. To explore the function of GGPPS in hepatic lipid homeostasis, we established liver-specific GGPPS knockout mice that fed with regular chow and high-fat diet (HFD). Mice with liver-specific GGPPS deletion were protected from HFD-induced hepatic steatosis. Further investigation revealed that GGPPS deficiency altered the ratio of FPP to GGPP. The accumulated FPP resulted in the inhibition on de novo lipogenesis (DNL) in hepatocytes, on one hand,

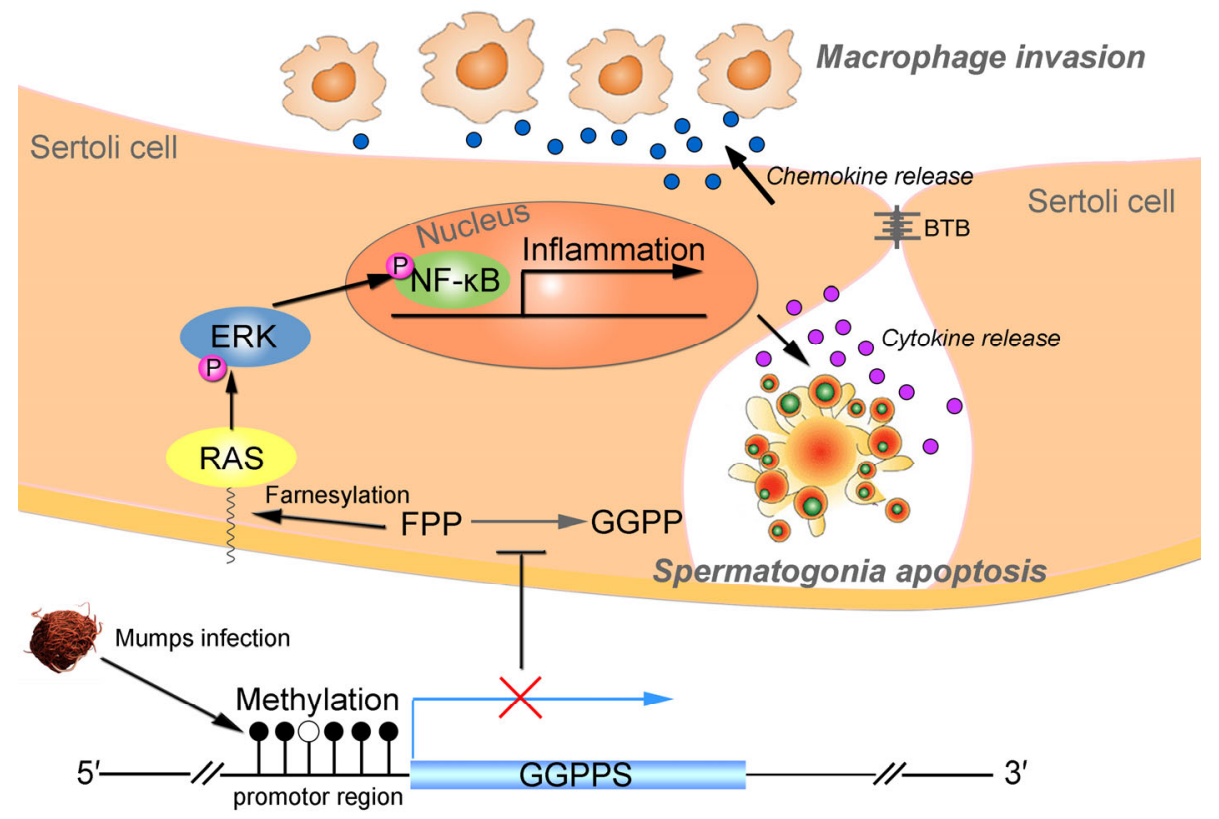

Figure 4 (color online) Mumps infection during childhood results in adult sterility through altered protein prenylation in Sertoli cells. The expression of GGPPS was decreased due to elevated promoter methylation in the testes of infertile patients with mumps infection history. GGPPS deletion in mouse Sertoli cells enhanced H-Ras farnesylation and constitutively activated MAPK Erk1/2, which was responsible for NF- $\kappa$ B activation and inflammatory reaction, and thus for the augmentation of cytokines and chemokines. These inflammatory factors then bound to the receptors on spermatogonia. Macrophages could be recruited by chemokines, easily invade the testicular tubules and attack developing spermatogonium cells. These result in spermatogonia apoptosis, leading to a long-term effect on adult infertility. 
through activating FXR/SHP signaling which in turn suppressed SREBP-1 expression and processing, and LXR activation, as reported elsewhere [42]; on the other hand, the inhibition is through promoting the LKB1/AMPK signaling (unpublished data). All in all, GGPPS is a key regulator of lipid metabolism in the liver. Specific targeting GGPPS in the liver may provide a valuable therapeutic strategy for hepatic steatosis.

\subsection{Protein prenylation and cardiac hypertrophy}

Growing evidence has suggested that small GTPases family (including Ras and Rho) and heterotrimeric G proteins are activated by a variety of extracellular ligands, such as angiotension II, endothelin-1, phenylephrine, as well as mechanical forces, which can lead to cardiac hypertrophy mediated by GPCR [43-45]. Hypercholesterolemia is a key cause for atherosclerosis and associated cardiovascular pathologies, including thrombosis and infarction. Statins, the HMG-CoA reductase inhibitors, often have beneficial effects on these symptoms by efficiently lowering cholesterol and the prenylation of RhoA in either clinical trials or animal models research [46]. Genetic disruption of Rce-1 in mice led to a dilated cardiomyopathy [47]. Furthermore, cardiac-specific overexpression of FPPS induced cardiac hypertrophy and dysfunction through RhoA hyperactivation [48]. Chronic inhibition of FPPS attenuated cardiac hypertrophy in spontaneously hypertensive rats [49]. Early in 2006, Olson's group has indicated that the enzymes involved in the mevalonate pathway, including HMG-CoA reductase and GGPPS, controlled the heart formation in Drosophila [50]. From the above research, we proposed that there might be a balance between protein geranylgeranylation and farnesylation that is required for heart function maintenance.

GGPPS, which is a branch point enzyme in the mevalonate metabolic pathway, can utilize FPP to synthesize
GGPP. Previous studies have suggested that treatment with DGBP could accumulate the level of FPP and increase protein farnesylation [18]. Therefore, we thought that GGPPS might be important for heart function as a critical switch to regulate these two protein prenylation. The 2013 statistical update data from American Heart Association showed that adjusted population attributable fraction for cardiovascular disease mortality was $40.6 \%$ for high blood pressure [51]. Our data suggested that GGPPS was downregulated in the hearts after pressure overload and in failing human hearts. Cardiac-specific GGPPS knockout mice died around two months old owing to severe heart failure, characterized by cardiac fibrosis, apoptosis and decreased heart function. Further analysis indicated that GGPPS deletion in cardiomyocytes led to the disrupted balance between protein farnesylation and geranylgeranylation. Increased Rheb farnesylation overactivated mTORC1 signaling and induced cardiomyocyte hypertrophy. The persistent cardiomyocyte hypertrophy caused the cardiac function to decrease with aging, which ultimately led to heart failure [52]. In conclusion, our data demonstrated that GGPPS played an indispensable role in postnatal heart function maintenance. The disrupted balance of protein prenylation by GGPPS ablation became a main cause for pathological heart remodeling (Figure 5). In the clinic, the inhibition of protein farnesylation or drugs that properly increase the level of geranylgeranylation provided new insight into pathologically hypertrophic heart diseases.

\section{Future directions}

GGPPS, a key enzyme for GGPP synthesis, can utilize FPP to produce GGPP. The geranylgeranylation of small G proteins regulated by GGPPS promotes the protein-membrane interaction and transduces the downstream signaling path-

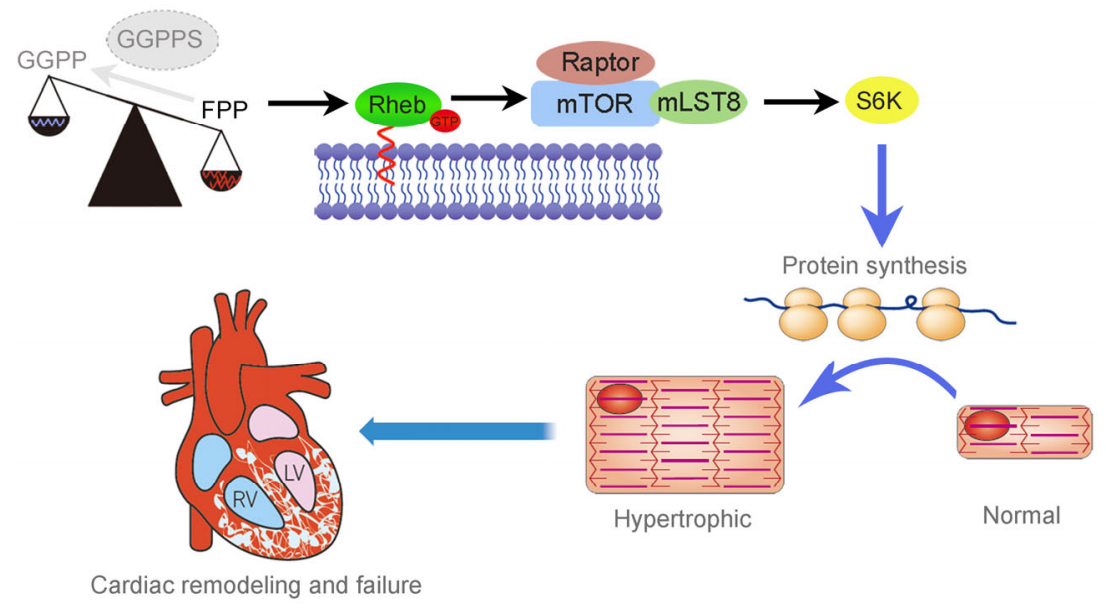

Figure 5 (color online) Disrupted protein prenylation balance regulates cardiomyocyte hypertrophy and chronic heart failure. GGPPS deficiency in cardiomyocytes disrupted the balance between protein farnesylation and geranylgeranylation. FPP accumulation increased Rheb farnesylation and then overactivated mTORC1 signaling pathway, which induced cardiomyocyte hypertrophy. The persistent cardiomyocyte hypertrophy impaired cardiac function and induced pathological cardiac remodeling, which ultimately led to heart failure. 
way. Disturbed GGPPS will lead to FPP accumulation and increase protein farnesylation, and thus activate the totally different signaling pathway. More than 100 proteins necessary for eukaryotic cell growth and differentiation are experimentally proven to be posttranslationally modified by the covalent attachment of an isoprenoid lipid [53]. However, 587 human genes encoding proteins provided from UniProt database possess a CXXX motif at their C-terminal end, which suggests that a large number of modified proteins need to be identified. Current technological methods to characterize protein prenylation are for the most part to study individual proteins. Therefore, to establish more sophisticated techniques that are capable of analyzing prenylation on a global scale towards the entire prenylome and uncover each concrete disease will be a great challenge in future research.

Besides, GGPPS can precisely control all kinds of physiological and pathological functions through interactions with other proteins. Growing evidence suggests that GGPP and FPP can directly bind to nuclear receptors (such as LXR, FXR, RXR) as a ligand to activate or suppress the role of transcriptional coactivators, and then regulate the expression of downstream proteins [54,55]. FPP was reported to promote osteoblast differentiation by enhancing FXR activity [56], while GGPP, as a LXR inhibitor, was involved in regulating cholesterol production and lipogenesis response to glucose concentration [57,58]. Taken together, GGPPS can affect the levels of GGPP and FPP, and may widely regulate the cellular physiology and pathology via a protein prenylation-independent pathway.

This work was supported by the National Basic Research Program of China (2012CB524900, 2009CB918703) and National Natural Science Foundation of China (31271540, 30671086) awarded to Li ChaoJun.

1 McTaggart S. Isoprenylated proteins. Cell Mol Life Sci, 2006, 63: 255-267

2 Perez-Sala D. Protein isoprenylation in biology and disease: general overview and perspectives from studies with genetically engineered animals. Front Biosci, 2007, 12: 4456-4472

3 Rodríguez-Concepción $\mathrm{M}$, Boronat A. Elucidation of the methylerythritol phosphate pathway for isoprenoid biosynthesis in bacteria and plastids. A metabolic milestone achieved through genomics. Plant Physiol, 2002, 130: 1079-1089

4 Eisenreich W, Bacher A, Arigoni D, Rohdich F. Biosynthesis of isoprenoids via the non-mevalonate pathway. Cell Mol Life Sci, 2004, 61: $1401-1426$

5 Vranová E, Coman D, Gruissem W. Network analysis of the MVA and MEP pathways for isoprenoid synthesis. Ann Rev Plant Biol, 2013, 64: 665-700

6 Maurer-Stroh S, Washietl S, Eisenhaber F. Protein prenyltransferases. Genome Biol, 2003, 4: 212

7 Berndt N, Hamilton AD, Sebti SM. Targeting protein prenylation for cancer therapy. Nat Rev Cancer, 2011, 11: 775-791

8 Carboni JM, Yan N, Cox AD, Bustelo X, Graham SM, Lynch MJ, Weinmann R, Seizinger BR, Der CJ, Barbacid M. Farnesyltransferase inhibitors are inhibitors of ras but not r-ras2/tc21, transformation. Oncogene, 1995, 10: 1905-1913

9 Whyte DB, Kirschmeier P, Hockenberry TN, Nunez-Oliva I, James L,
Catino JJ, Bishop WR, Pai J-K. K-and N-Ras are geranylgeranylated in cells treated with farnesyl protein transferase inhibitors. J Biol Chem, 1997, 272: 14459-14464

10 Downward J. Targeting RAS signalling pathways in cancer therapy. Nat Rev Cancer, 2003, 3: 11-22

11 Mijimolle N, Velasco J, Dubus P, Guerra C, Weinbaum CA, Casey PJ, Campuzano V, Barbacid M. Protein farnesyltransferase in embryogenesis, adult homeostasis, and tumor development. Cancer Cell, 2005, 7: 313-324

12 Sjogren AK, Andersson KM, Liu M, Cutts BA, Karlsson C, Wahlstrom AM, Dalin M, Weinbaum C, Casey PJ, Tarkowski A, Swolin B, Young SG, Bergo MO. GGTase-I deficiency reduces tumor formation and improves survival in mice with K-Ras-induced lung cancer. J Clin Invest, 2007, 117: 1294-1304

13 Khan OM, Ibrahim MX, Jonsson IM, Karlsson C, Liu M, Sjogren AK, Olofsson FJ, Brisslert M, Andersson S, Ohlsson C, Hulten LM, Bokarewa M, Bergo MO. Geranylgeranyltransferase type I (GGTase-I) deficiency hyperactivates macrophages and induces erosive arthritis in mice. J Clin Invest, 2011, 121: 628-639

14 Shotelersuk V, Gahl WA. Hermansky-pudlak syndrome: models for intracellular vesicle formation. Mol Genet Metab, 1998, 65: 85-96

15 Larijani B, Hume AN, Tarafder AK, Seabra MC. Multiple factors contribute to inefficient prenylation of Rab27a in Rab prenylation diseases. J Biol Chem, 2003, 278: 46798-46804

16 Pereira-Leal JB, Hume AN, Seabra MC. Prenylation of rab GTPases: molecular mechanisms and involvement in genetic disease. FEBS Lett, 2001, 498: 197-200

17 Weivoda MM, Hohl RJ. Geranylgeranyl pyrophosphate stimulates PPAR $\gamma$ expression and adipogenesis through the inhibition of osteoblast differentiation. Bone, 2012, 50: 467-476

18 Weivoda MM, Hohl RJ. The effects of direct inhibition of geranylgeranyl pyrophosphate synthase on osteoblast differentiation. J Cell Biochem, 2011, 112: 1506-1513

19 Li CJ, Ning W, Matthay MA, Feghali-Bostwick CA, Choi AM. MAPK pathway mediates EGR-1-HSP70-dependent cigarette smoke-induced chemokine production. Am J Physiol-Lung Cell Mol Physiol, 2007, 292: L1297-1303

20 Ning W, Dong Y, Sun J, Li C, Matthay MA, Feghali-Bostwick CA, Choi AM. Cigarette smoke stimulates matrix metalloproteinase-2 activity via EGR-1 in human lung fibroblasts. Am J Resp Cell Mol Biol, 2007, 36: 480

21 Ning W, Li CJ, Kaminski N, Feghali-Bostwick CA, Alber SM, Di YP, Otterbein SL, Song R, Hayashi S, Zhou Z, Pinsky DJ, Watkins SC, Pilewski JM, Sciurba FC, Peters DG, Hogg JC, Choi AM. Comprehensive gene expression profiles reveal pathways related to the pathogenesis of chronic obstructive pulmonary disease. Proc Natl Acad Sci USA, 2004, 101: 14895-14900

22 Shen N, Shao Y, Lai SS, Qiao L, Yang RL, Xue B, Pan FY, Chen HQ, Li CJ. GGPPS, a new EGR-1 target gene, reactivates ERK 1/2 signaling through increasing ras prenylation. Am J Pathol, 2011, 179: 2740-2750

23 Chen H, Wang L, Gong T, Yu Y, Zhu C, Li F, Wang L, Li C. EGR-1 regulates Ho-1 expression induced by cigarette smoke. Biochem Biophys Res Commun, 2010, 396: 388-393

24 Shen N, Gong T, Wang JD, Meng FL, Qiao L, Yang RL, Xue B, Pan FY, Zhou XJ, Chen HQ, Ning W, Li CJ. Cigarette smoke-induced pulmonary inflammatory responses are mediated by EGR-1/ GGPPS/MAPK signaling. Am J Pathol, 2011, 178: 110-118

25 Hotamisligil GS. Inflammation and metabolic disorders. Nature, 2006, 444: 860-867

26 Vicent D, Maratos-Flier E, Kahn CR. The branch point enzyme of the mevalonate pathway for protein prenylation is overexpressed in the ob/ob mouse and induced by adipogenesis. Mol Cell Biol, 2000, 20: 2158-2166

27 Solomon C, Leitner J, Goalstone M. Dominant negative $\alpha$-subunit of farnesyl-and geranylgeranyl-transferase I inhibits insulin-induced differentiation of 3T3-L1 pre-adipocytes. Int J Obes Relat Metab Disord, 2003, 27: 40-47

28 Shen N, Yu X, Pan FY, Gao X, Xue B, Li CJ. An early response 
transcription factor, EGR-1, enhances insulin resistance in type 2 diabetes with chronic hyperinsulinism. J Biol Chem, 2011, 286: 14508-14515

29 Yu X, Shen N, Zhang ML, Pan FY, Wang C, Jia WP, Liu C, Gao Q, Gao X, Xue B, Li CJ. EGR-1 decreases adipocyte insulin sensitivity by tilting PI3K/Akt and MAPK signal balance in mice. EMBO J, 2011, 30: 3754-3765

30 Brennan J, Capel B. One tissue, two fates: molecular genetic events that underlie testis versus ovary development. Nat Rev Genet, 2004, 5: 509-521

31 Mackay S. Gonadal development in mammals at the cellular and molecular levels. Int Rev Cytol, 2000, 200: 47-99

32 Philip J, Selvan D, Desmond AD. Mumps orchitis in the non-immune postpubertal male: a resurgent threat to male fertility? BJU Int, 2006, 97: $138-141$

33 Setchell BP. Blood-testis barrier, junctional and transport proteins and spermatogenesis. Adv Exp Med Biol, 2008, 636: 212-233

34 Petersen C, Söder O. The sertoli cell-a hormonal target and 'super'nurse for germ cells that determines testicular size. Horm Res Paediatr, 2006, 66: 153-161

35 Sun B, Qi N, Shang T, Wu H, Deng T, Han D. Sertoli cell-initiated testicular innate immune response through toll-like receptor-3 activation is negatively regulated by Tyro3, Axl, and mer receptors. Endocrinology, 2010, 151: 2886-2897

36 Riccioli A, Starace D, Galli R, Fuso A, Scarpa S, Palombi F, De Cesaris P, Ziparo E, Filippini A. Sertoli cells initiate testicular innate immune responses through TLR activation. J Immunol, 2006, 177: $7122-7130$

37 Wang XX, Ying P, Diao F, Wang Q, Ye D, Jiang C, Shen N, Xu N, Chen WB, Lai SS, Jiang S, Miao XL, Feng J, Tao WW, Zhao NW, Yao B, Xu ZP, Sun HX, Li JM, Sha JH, Huang XX, Shi QH, Tang H, Gao X, Li CJ. Altered protein prenylation in sertoli cells is associated with adult infertility resulting from childhood mumps infection. J Exp Med, 2013, 210: 1559-1574

38 Frith J, Day CP, Robinson L, Elliott C, Jones DE, Newton JL. Potential strategies to improve uptake of exercise interventions in non-alcoholic fatty liver disease. J Hepatol, 2010, 52: 112-116

39 Wang L, Shen ZH, Xu Q. Study on the prevalence of nonalcoholic fatty liver and the association between nonalcoholic fatty liver and metabolic syndrome. Chin J Convalesc Med, 2010, 5: 067

40 Kotronen A, Westerbacka J, Bergholm R, Pietiläinen KH, Yki-Järvinen H. Liver fat in the metabolic syndrome. J Clin Endocrinol Metab, 2007, 92: 3490-3497

41 Neuschwander-Tetri BA, Caldwell SH. Nonalcoholic steatohepatitis: summary of an AASLD single topic conference. Hepatology, 2003, 37: 1202-1219

42 Watanabe M, Houten SM, Wang L, Moschetta A, Mangelsdorf DJ, Heyman RA, Moore DD, Auwerx J. Bile acids lower triglyceride levels via a pathway involving FXR, SHP, and SREBP-1c. J Clin Invest, 2004, 113: 1408-1418

43 Hunter J, Tanaka N, Rockman H, Ross J, Chien K. Ventricular expression of a MLC-2v-ras fusion gene induces cardiac hypertrophy and selective diastolic dysfunction in transgenic mice. J Biol Chem, 1995, 270: 23173
44 Sah VP, Minamisawa S, Tam SP, Wu TH, Dorn GW, Ross J, Chien $\mathrm{KR}$, Brown JH. Cardiac-specific overexpression of RhoA results in sinus and atrioventricular nodal dysfunction and contractile failure. J Clin Invest, 1999, 103: 1627-1634

45 Heineke J, Molkentin J. Regulation of cardiac hypertrophy by intracellular signalling pathways. Nat Rev Mol Cell Biol, 2006, 7: 589-600

46 Porter KE, Turner NA. Statins and myocardial remodelling: cell and molecular pathways. Expert Rev Mol Med, 2011, 13: e22

47 Bergo MO, Lieu HD, Gavino BJ, Ambroziak P, Otto JC, Casey PJ, Walker QM, Young SG. On the physiological importance of endoproteolysis of CAAX proteins. J Biol Chem, 2004, 279: 4729

48 Yang J, Mou Y, Wu T, Ye Y, Jiang JC, Zhao CZ, Zhu HH, Du CQ, Zhou L, Hu SJ. Cardiac-specific overexpression of farnesyl pyrophosphate synthase induces cardiac hypertrophy and dysfunction in mice. Cardiovasc Res, 2013, 97: 490-499

49 Li L, Chen G, Yang Y, Ye Y, Yao L, Hu S. Chronic inhibition of farnesyl pyrophosphate synthase attenuates cardiac hypertrophy and fibrosis in spontaneously hypertensive rats. Biochem Pharmacol, 2010, 79: 399-406

50 Yi P, Han Z, Li X, Olson E. The mevalonate pathway controls heart formation in Drosophila by isoprenylation of Ggamma 1. Science, 2006, 313: 1301

51 Go AS, Mozaffarian D, Roger VL, Benjamin EJ, Berry JD, Borden WB, Bravata DM, Dai S, Ford ES, Fox CS, Franco S, Fullerton HJ, Gillespie C, Hailpern SM, Heit JA, Howard VJ, Huffman MD, Kissela BM, Kittner SJ, Lackland DT, Lichtman JH, Lisabeth LD, Magid D, Marcus GM, Marelli A, Matchar DB, McGuire DK, Mohler ER, Moy CS, Mussolino ME, Nichol G, Paynter NP, Schreiner PJ, Sorlie PD, Stein J, Turan TN, Virani SS, Wong ND, Woo D, Turner MB, American Heart Association Statistics C, Stroke Statistics S. Executive summary: heart disease and stroke statistics2013 update: a report from the american heart association. Circulation, 2013, 127: 143-152

52 Xu N, Guan S, Chen Z, Yu Y, Xie J, Pan FY, Zhao NW, Liu L, Yang $\mathrm{ZZ}$, Gao X, Xu B, Li CJ. The alteration of protein prenylation induces cardiomyocyte hypertrophy through Rheb-mTORC1 signalling and leads to chronic heart failure. J Pathol, 2014, doi: 10.1002/path.4480

53 Lane KT, Beese LS. Thematic review series: lipid posttranslational modifications. Structural biology of protein farnesyltransferase and geranylgeranyltransferase type I. J Lipid Res, 2006, 47: 681-699

54 Edwards PA, Kast HR, Anisfeld AM. Bareing it all: the adoption of LXR and FXR and their roles in lipid homeostasis. J Lipid Res, 2002, 43: $2-12$

55 Kalaany NY, Mangelsdorf DJ. LXRS and FXR: The yin and yang of cholesterol and fat metabolism. Ann Rev Physiol, 2006, 68: 159-191

56 Id Boufker H, Lagneaux L, Fayyad-Kazan H, Badran B, Najar M, Wiedig M, Ghanem G, Laurent G, Body JJ, Journé F. Role of farnesoid $\mathrm{X}$ receptor (FXR) in the process of differentiation of bone marrow stromal cells into osteoblasts. Bone, 2011, 49: 1219-1231

57 Bełtowski J. Liver $\mathrm{X}$ receptors (LXR) as therapeutic targets in dyslipidemia. Cardiovasc Ther, 2008, 26: 297-316

58 Mitro N, Mak PA, Vargas L, Godio C, Hampton E, Molteni V, Kreusch A, Saez E. The nuclear receptor LXR is a glucose sensor. Nature, 2006, 445: 219-223

Open Access This article is distributed under the terms of the Creative Commons Attribution License which permits any use, distribution, and reproduction in any medium, provided the original author(s) and source are credited. 